\title{
Myocardial tagging for the analysis left ventricular function
}

\author{
Eike Nagel $^{\mathrm{a}, \mathrm{b}, *}$, Matthias Stuber ${ }^{\mathrm{c}}$, E. Fleck ${ }^{\mathrm{d}}$, Peter Boesiger ${ }^{\mathrm{c}}$, Otto M. Hess ${ }^{\mathrm{a}}$

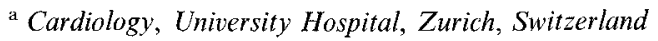 \\ ' Deutsches Herzzentrum Berlin, Medizinische Klinik, Abteilung für Kardiologie, Augustenburger Platz 1, 13353 Berlin, Germany \\ 'Institute of Biomedical Engineering and Medical Informatics, University and Federal Institute of Technology, Zurich, Switzerland \\ 'Internal Medicine/Cardiology, Charité, Campus Virchow, Humboldt University and Deutsches Herzzentrum, Berlin, Germany
}

Keywords: Myocardial tagging; 3D-motion; Heart

\section{Introduction}

The 3D-motion of the heart is complex and can be described by 3 different motion components, i.e. radial displacement, rotation and translation (Fig. 1). Alterations in 3D-motion have been reported in LV hypertrophy, cardiomyopathy and in patients with myocardial ischemia and infarction. Relaxation abnormalities are one of the most sensitive markers for detection of changes in myocardial energy level (e.g. ischemia) or structure (e.g. hypertrophy). In the following systolic and diastolic 3D-motion of the left ventricle is discussed in patients after myocardial infarction and patients with aortic stenosis using MR myocardial tagging.

Myocardial tagging is a non invasive technique based on magnetic resonance which allows to label the myocardium by spatial modulation of magnetization e.g. with a rectangular grid (Fig. 2). These tags are fixed on the myocardium during systolic contraction and diastolic relaxation. From the distortion of the grid and the displacement of the grid-crossing points regional shortening as well as the rotational motion of the right and left ventricle can be assessed in different projections, e.g. basal, equatorial, and apical short-axis views. An improvement of the original SPAMM technique was achieved by the use of several modifications (CSPAMM, Complementary SPAtial Modulation of

\footnotetext{
* Corresponding author.
}

Magnetization). Tag persistence was prolonged to more than $1000 \mathrm{~ms}$ by the use of two measurements with complementary tagging signs which were subtracted. Tag contrast was increased by the use of variable flip angles for imaging. Through plane motion was corrected for by a slice-following technique. Imaging time was reduced by the combination with an echoplanar imaging technique which allowed to use breath holding to suppress respiratory artifacts.

With these improvements the analysis of one full cardiac cycle with high temporal and spatial resolution within a single breath hold is possible.

\section{Image acquisition}

CSPAMM was used on a conventional $1.5 \mathrm{~T}$ magnetic resonance system (Gyroscan NT, Philips, Best, The Netherlands) in prone position using a cardiac surface coil. An ECG was recorded and respiratory motion was checked with a strain gauge. After two short scans for the localization of the longitudinal heart axis, 3 short-axis planes (basal, $1 \mathrm{~cm}$ below the valvular annulus; equatorial, mid-distance between basal and apical plane; apical, $1 \mathrm{~cm}$ within the RV cavity) were imaged and labeled with a rectangular grid (spacing 8 $\mathrm{mm})$. A total of 16 images was acquired for each imaging plane beginning at end-diastole $(24 \mathrm{~ms}$ after peak $\mathrm{R}$-wave) and ending with the next end-diastole. Temporal resolution was $35 \mathrm{~ms}$ and spatial resolution 


\section{D-motion of the heart}

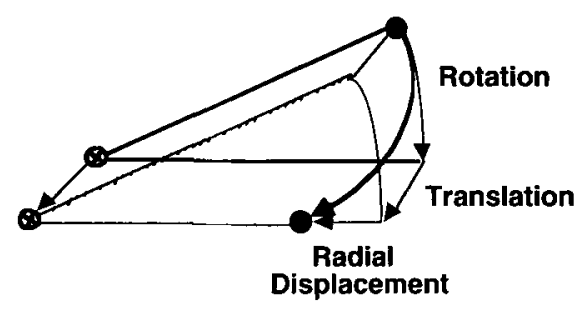

- Center of Gravity

Fig. 1. 3-D cardiac motion.

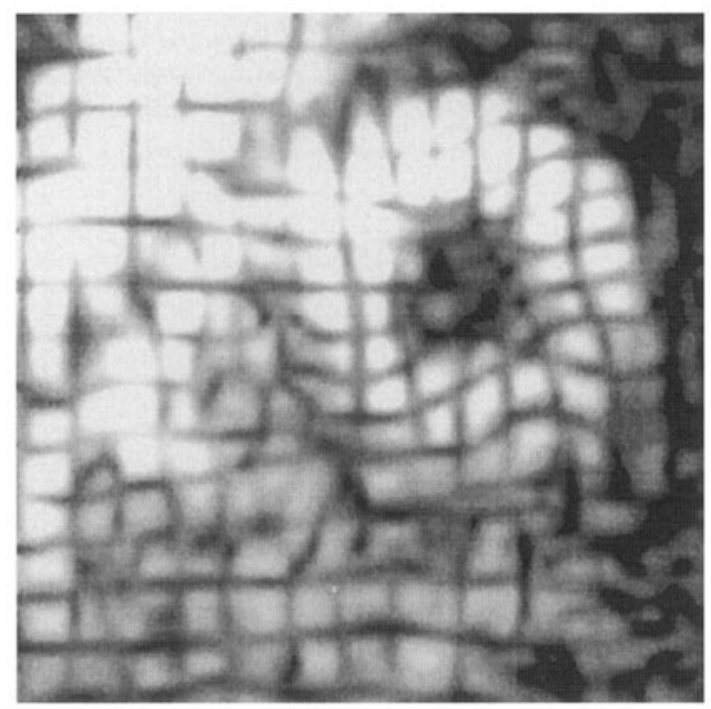

Fig. 2. Short axis view. A rectangular tagging grid was placed on the myocardium at end-diastole. Please note the distortion of the grid at end-systole.

$2.3 \times 2.3 \mathrm{~mm}^{2}$ with a slice thickness of $6 \mathrm{~mm}$ at the apex, $7 \mathrm{~mm}$ at the equator and $8 \mathrm{~mm}$ at the base. Images were acquired during a breath hold of 16-20 $\mathrm{s}(\mathrm{TE}=7.2 \mathrm{~ms}, \mathrm{TR}=800 \mathrm{~ms}$, EPI-factor 3, field of view $300 \times 300 \mathrm{~mm}^{2}$ ). Two sets of images were acquired for tagging in the horizontal and vertical direc- tion. A rectangular grid was achieved by multiplication of the two images.

\section{Image analysis}

The intersection points of the tagging lines were marked and traced semiautomatically in each image using a custom-written evaluation program on a DEC alpha work station. Epi- and endocardial borders were defined manually in the first image and automatically determined in all other images using the motion of the grid crossing points. End-diastole was defined as the first image after the R-wave, end-systole as the image with the smallest cavity volume.

\section{Definition of terms}

Rotation angular displacement of the gridcrossing points around the center of gravity

Radial displacement of the grid-crossing displacement points towards the center of gravity Translation longitudinal displacement of the heart Wringing rotation of the base with opposite romotion tation of the apex

Twisting systolic rotation of the heart Untwisting diastolic rotation of the heart Area change fractional change of the luminal area Circumferential percent change of the distance beshortening tween two midmyocardial points

\section{3D-motion of the normal left ventricle}

The normal left ventricle performs a systolic clockwise rotation at the base $\left(-3 \pm 2^{\circ}\right)$ and a counterclockwise rotation at the apex $\left(+12 \pm 4^{\circ}\right)$ which occurs mainly during isovolumic contraction and results in a systolic 'wringing' motion (Fig. 3). The

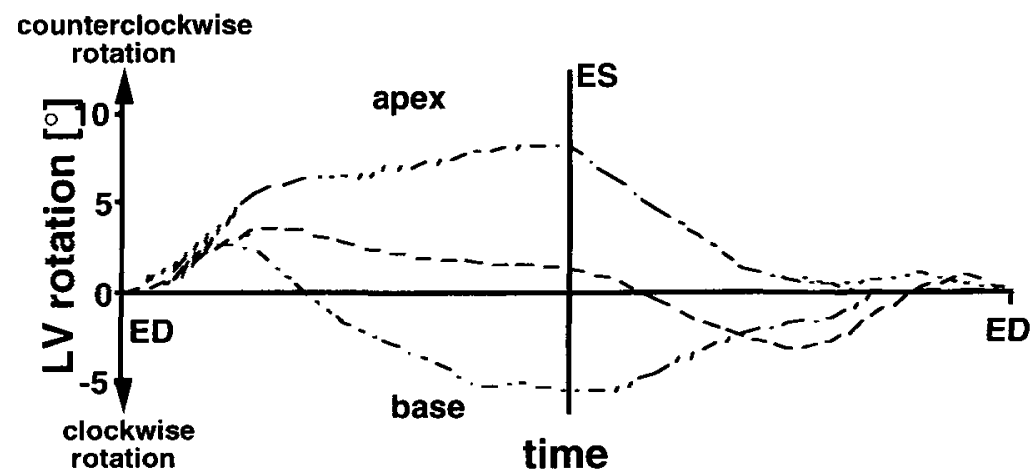

Fig. 3. Wringing motion. A clockwise rotation of the cardiac base and a counterclockwise rotation of the apex occur during systolic contraction, resulting in a wringing motion of the left ventricle. 
'wringing' motion of the left ventricle allows the heart to achieve a high intracavitary pressure with minimal radial displacement (analogous to the 'wringing' of a wet towel). An 'untwisting' motion which precedes diastolic filling is observed during isovolumic relaxation which is directed opposite to the systolic "wringing' motion and results in a counterclockwise rotation at the base and a clockwise rotation at the apex. It is an important determinant of elastic recoil and thus, of diastolic filling and suction. During the systolic ejection and diastolic filling phase hardly any rotation occurs.

\section{3D-motion of the left ventricle after myocardial in- farction}

Regional motion in myocardial infarction depends largely on the loacalization of the infarct and is typically altered with a loss of regional shortening and a prolongation of the 'untwisting' process with a consecutive reduction in relaxation and diastolic filling. In 14 patients with anterolateral infarcts $L V$ area reduction was reduced in infarct regions $(23 \pm 11 \%)$ when compared to normal regions $(35 \pm 10 \%, P<0.05)$. At the same time a reduction of regional circumferential shortening of infarcted $(10 \pm 5 \% ; P<0.05$ vs remote and controls) and remote regions $(15 \pm 6 \%, P<0.05$ vs controls) in comparison to controls $(21 \pm 5 \%)$. In addition a prolongation of the 'untwisting' motion with a reduction in rotation velocity was observed.
7. 3D-motion of the left ventricle in patients with aortic stenosis

In 15 patients with aortic stenosis systolic rotation was reduced at the base $\left(-2 \pm 2^{\circ} ; P<0.05\right)$ but increased at the apex $\left(+15 \pm 6^{\circ} ; P<0.05\right)$. Diastolic untwisting was delayed and prolonged with a decrease in rotation velocity $\left(-7 \pm 1^{\circ} \mathrm{s}^{-1}\right)$ when compared to controls $\left(-11 \pm 2^{\circ} \mathrm{s}^{-1} ; P<0.001\right)$.

\section{Conclusions}

Based on our measurements following observations were made:

(1) The left ventricle performs a systolic wringing motion which occurs mainly during isovolumic contraction.

(2) Diastolic untwisting is found predominantly during isovolumic relaxation and occurs opposite to systolic rotation.

(3) After myocardial infarction regional shortening is reduced in infarcted and remote regions. Predominantly diastolic untwisting is delayed and prolonged.

(4) In patients with aortic stenosis apical rotation is enhanced, whereas diastolic untwisting is significantly inhibited, which explains the diastolic dysfunction in these patients.

Myocardial tagging makes an accurate regional wall motion analysis and the assessment of cardiac rotation possible and, thus, allow new insight into the mechanical function of the heart. 